\title{
Utilization of Rayleigh Waves for Engine Oil Level Measurements
}

\author{
P. Š́fer ${ }^{a}$, M. Š́fer ${ }^{b}$, J. Gebauer $^{a}$, P. Niegodajew ${ }^{c}$ And K. GruszkA ${ }^{d}$ \\ ${ }^{a}$ Department of Control Systems and Instrumentation, Faculty of Mechanical Engineering, \\ Vঙ̆B - Technical University of Ostrava, \\ 17. listopadu 15/2127, 70833 Ostrava-Poruba, Czech Republic \\ ${ }^{b}$ Department of Applied Mechanics, Faculty of Mechanical Engineering, \\ Vঙ̆B - Technical University of Ostrava, \\ 17. listopadu 15/2127, 70833 Ostrava-Poruba, Czech Republic \\ ${ }^{c}$ Department of Thermal Machinery, Czestochowa University of Technology, \\ Faculty of Mechanical Engineering and Computer Science, \\ Armii Krajowej 21, 42-201 Czestochowa, Poland \\ ${ }^{d}$ Faculty of Production Engineering and Materials Technology, \\ Częstochowa University of Technology, Armii Krajowej 19, 42-200 Częstochowa, Poland
}

Doi: $10.12693 /$ APhysPolA.138.272

*e-mail: pavel.sofer@vsb.cz

\begin{abstract}
The paper deals with the problematics of the measurement of engine oil level based on the correlation between the attenuation of the surface Rayleigh wave and the fluid level. Available literature offers a few methods, which utilizes the surface acoustic waves for such a purpose, leaving the problem not completely resolved. The theoretical assumptions regarding Rayleigh wave formation on the free surface were supported by numerical simulations in COMSOL Multiphysics software. The final part of this article describes an experimental device that has been designed and manufactured for the measurement purposes including the proposal of the measuring method based on the attenuation of Rayleigh surface waves under the influence of propagation along the solid-liquid interface.
\end{abstract}

topics: surface acoustic wave, FEM, fluid level measurement

\section{Introduction}

The proposed paper is focused on the possibility of measuring the level of engine oil with the use of the surface waves. The approach is based on the dependence of the surface wave amplitude on the measured height. It originates from the following principles: The emitted longitudinal wave within the wedge is hitting the wedge/prism interface, where under certain conditions in terms of the angle of incidence, the surface wave is being generated. An important condition for the formation of a surface wave, considering a particular excitation method, is the existence of a surface wave for a given angle of incidence relative to the surface of the functional part of the prism. Then, above mentioned, ultrasonic wedges can be manufactured with fixed or variable angle of incidence. Generated in such a way the surface wave propagates through an aluminum prism to its end, where it is reflected and reciprocally converted into longitudinal wave, again, using the wedge. Afterwards, it is possible to estimate the amplitude ratio between the incident and reflected wave. When a surface wave passes through the solid-liquid interface, the wave interacts with the liquid layer and some of its energy radiates into the liquid in the form of a volumetric longitudinal wave. The larger the interface, along which the

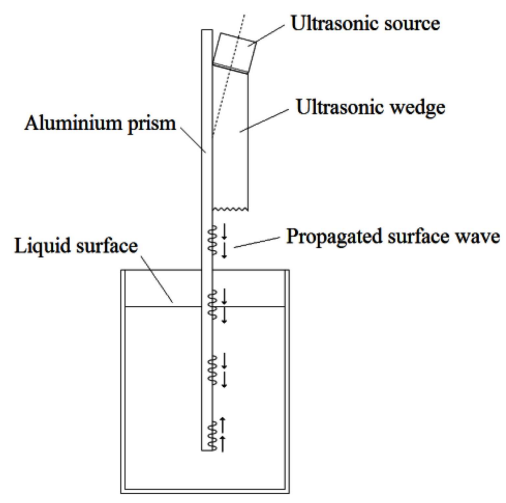

Fig. 1. Schematic description of the test stand.

wave radiates its energy into the liquid layer, the smaller amount of the energy will be transmitted through the solid waveguide and, at the same time, the smaller amplitude of the reflected wave will be registered. Figure 1 shows a schematic description of the test stand.

\section{Rayleigh surface waves}

The discovery of the Rayleigh waves including the mathematical description is associated with the early $19^{\text {th }}$ century, when this type of waves 


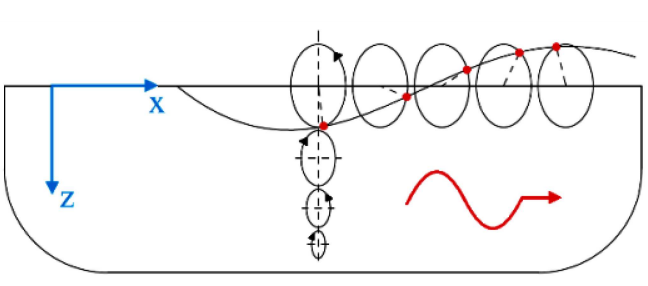

Fig. 2. Rayleigh wave propagating along the boundary of the half-space.

has been described by the English physicist Lord Rayleigh [1]. Rayleigh waves propagate along the free surface, where the wave penetration is approximately equal to one wavelength. Another interesting fact is that Rayleigh waves do not exhibit dispersion, i.e., the wave's phase/group velocity is not a function of its frequency $[2,3]$. Considering a wave propagation along the positive $x$ axis (see Fig. 2), the particles on the surface and in its close proximity are moving along elliptical orbits counter-clockwise, while from a certain depth a rotation will be reversed.

The identification of individual stress tensor and displacement vector components is realized with use of Helmholtz decomposition [4]. For the displacement vector $\bar{u}$, one applies:

$$
\bar{u}=\nabla \varphi+\nabla \times \bar{\psi}
$$

where $\varphi$ and $\bar{\psi}$ are the potential and the vector functions, respectively, defined as

$$
\varphi=\varphi(x, z) \quad \bar{\psi}=(0,-\psi(x, z), 0) .
$$

In case of the equation of motion expressed in terms of displacements

$$
\mu \nabla^{2} \bar{u}+(\lambda+\mu) \nabla(\nabla \bar{u})=\rho \ddot{\bar{u}}
$$

it can be further separated into two wave equations. One equation is for the dilatational waves and the other one for the shear waves, i.e.,

$$
\begin{aligned}
& \nabla^{2} \varphi-\frac{1}{c_{L}^{2}} \ddot{\varphi}=0, \\
& \nabla^{2} \psi-\frac{1}{c_{T}^{2}} \ddot{\psi}=0,
\end{aligned}
$$

where $\mu$ and $\lambda$ denote the Lame's constants, $\rho$ is the mass density per unit volume, $c_{L}$ refers to the wave speed of dilatational waves, $c_{T}$ is the wave speed of shear waves. Assuming the harmonic wave motion in the form of $\varphi=\Phi(z) \mathrm{e}^{\mathrm{i}(\omega t-k x)}$, $\psi=\Psi(z) \mathrm{e}^{\mathrm{i}(\omega t-k x)}$ and the following boundary conditions: $\sigma_{z}=\tau_{x z}=\tau_{z y}=0$, it is possible to derive, not only the expressions for the individual stress tensor and displacement vector components, but also the Rayleigh wave equation. Moreover, one can calculate the speed of Rayleigh wave in a given medium:

$$
\alpha_{S}^{6}-8 \alpha_{S}^{4}+\left(24-16 \alpha_{T L}^{2}\right) \alpha_{S}^{2}+16 \alpha_{T L}^{2}-16=0,
$$

where

$$
\alpha_{T L}^{2}=\frac{c_{T}^{2}}{c_{L}^{2}}=\frac{1-2 \nu}{2-2 \nu},
$$

$$
\alpha_{S}^{2}=\frac{c_{R}^{2}}{c_{T}^{2}}=\frac{k_{T}^{2}}{k_{e}^{2}} .
$$

Here, $c_{R}$ is the Rayleigh wave speed, $k$ refers to the wavenumber of Rayleigh wave, $k_{T}$ is the wavenumber of shear waves, while $\nu$ is the Poisson's ratio $[5,6]$.

\section{Numerical simulation}

The aim of realized simulations was to verify the theoretical assumptions of the Rayleigh surface wave formation in considered geometry. If a longitudinal ultrasonic wave hits the interface between two environments with different acoustic impedances, refraction, reflection, or transformation to another type of wave may occur. Relations between the angles and velocities are given by the so-called Snell's law $[7,8]$. The plexiglass wedge was considered for the transfer of acoustic energy from the source of oscillations to the prism.

The simulation did not consider the real interface between the wedge and the steel prism. In this case, the ideal contact between both geometries has been defined. To create a Rayleigh surface wave, the driving longitudinal wave from the source must be hitting the plexiglass/steel interface under certain incident angle.

Figure 3 shows the course of the total displacement on the geometry under consideration at selected simulation times. The generated surface wave propagates over the free surface of the steel prism, where at time $t=50 \mu \mathrm{s}$, the wave is reflected from the free end of the prism.

The existence of the surface Rayleigh wave in the simulation was confirmed by the course of the individual components of the displacement vector in $x-y$ coordinates, measured at a selected point (Point sensor mark, see Fig. 4) on the surface of the steel prism. Elliptical trajectories were observed counterclockwise.

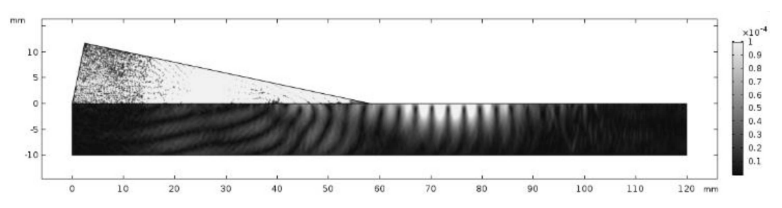

Fig. 3. Total displacement field (Sim. time: $35 \mu \mathrm{s}$, top to bottom).

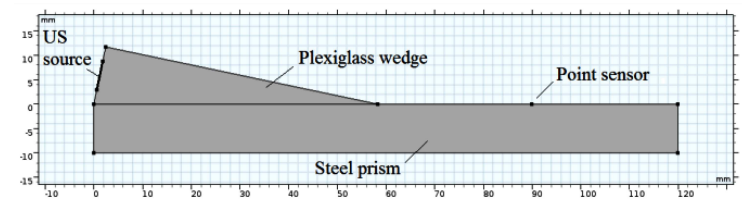

Fig. 4. Geometry considered in the numerical simulation. 


\section{Experimental measurements}

An experimental stand has been developed in order to comprehensively investigate the relation between the immersion depth of the prism and the surface wave amplitude. For the surface Rayleigh wave excitation has been used a wedge with predefined angle of incidence, which has been manufactured from extruded plexiglass of $20 \mathrm{~mm}$ thickness. Although a steel prism was considered in the simulations, an aluminum prism was used for measurements because of easy machinability and mechanical adjustments. The considered material change had no effect on the surface wave itself.

A two-channel Optel OPBOX V2.1 compact generator together with Olympus M109 $5 \mathrm{MHz}$ probe was used for generation and acquisition of ultrasonic waves. Castrol Edge 5W30 oil with room temperature of $25^{\circ} \mathrm{C}$ was used as a test liquid medium. The aluminum prism was immersed in the oil across a range of $0-40 \mathrm{~mm}$ in $4 \mathrm{~mm}$ increments, where for each configuration in terms of the immersion depth, multiple B-scans of the surface wave echo amplitude have been captured. One has to note, that in the initial position, which corresponds to the immersion depth of $0 \mathrm{~mm}$, was the oil level prism touching the free surface of the liquid.

Figures 5 and 6 display the obtained dependencies between the normalized wave amplitudes and the immersion depth. The measured mean values of the maximal amplitudes were then used for the design of the regression functions of first, second and third order and also exponential one.

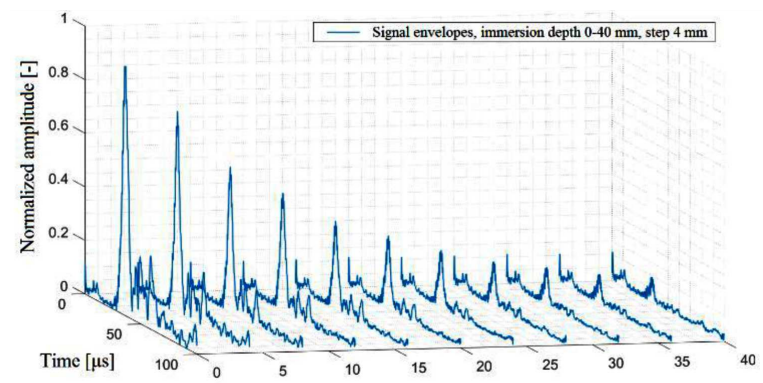

Fig. 5. Absolute amplitude signal envelopes corresponding to selected prism immersion depths.

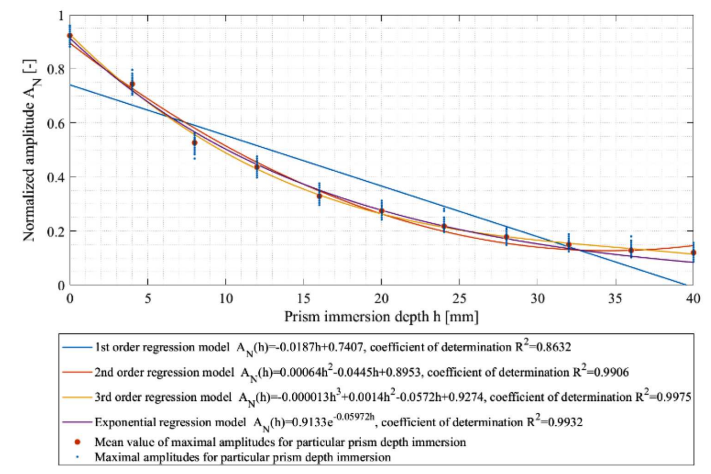

Fig. 6. Comparison of used regression models.

\section{Conclusions}

Polynomial regression models of the first, second and third order, as well as the exponential model were developed based on the normal distribution of individual samples. The individual models have been designed on the basis of the mean values of the maximum signal amplitudes (100 measurements) for each particular immersion depth. By applying higher-order polynomials, the regression curve approximates the experimentally measured points in better way, however, the model then describes to a greater extent the noise of the experimental data in itself, rather than the desired functional dependence. Akaike information criterion was chosen to select the appropriate regression model, where the optimal regression model is characterized by the minimum value of the AIC parameter (see Table I).

TABLE I

Evaluation of regression models based on Akaike criterion.

\begin{tabular}{c|c}
\hline \hline Regression model & AIC parameter \\
\hline 1st order polynomial & -42.513 \\
2nd order polynomial & -66.764 \\
3rd order polynomial & -74.013 \\
exponential function & -75.503
\end{tabular}

By comparing the AIC parameters, it can be stated, that the measured data best describes the exponential regression model. Thus, the decay of the signal amplitude exhibits exponential decay with the immersion depth of the prism.

\section{Acknowledgments}

This work was supported by the European Regional Development Fund in the Research Centre of Advanced Mechatronic Systems project, CZ.02.1.01/0.0/0.0/16 019/0000867 within the Operational Programme Research, Development and Education and the project SP2020/57 Research and Development of Advanced Methods in the Area of Machines and Process Control supported by the Ministry of Education, Youth and Sports.

Research work was also supported by the Ministry of Education, Youth and Sports project No. 8JPL19022 and NAWA project No. PPN/BIL/2018/1/00030.

\section{References}

[1] L. Rayleigh, Proc. London Math. Soc. s1-17, 4 (1885).

[2] M.H. Sadd, Wave Motion and Vibration in Continuous Media, University of Rhode Island, Kingston (RI) 1990. 
[3] J. Achenbach, Wave Propagation in Elastic Solids, Vol. 16, North-Holland Series in Applied Mathematics and Mechanics, North Holland, 1987.

[4] J. Bladel, "On Helmholtz's Theorem in Finite Regions", AEC research and development report no. MURA-440, U.S. Atomic Energy Commission, 1958.

[5] J.L. Rose, Ultrasonic Guided Waves in Solid Media, Cambridge University Press, 2014.
[6] I.A. Viktorov, Rayleigh and Lamb Waves: Physical Theory and Applications (Ultrasonic Technology), Springer, 2013.

[7] J. Obraz, Ultrazvuk $v$ měřici technice, SNTL - Nakladatelství technické literatury, Praha 1976 (in Czech).

[8] V. Sakharov, S. Kuznetsov, B. Zaitsev, I. Kuznetsova, S. Joshi, Ultrasonics 41, 319 (2003). 